\title{
Valoración del desempeño lumínico de puestos de trabajo a partir de los efectos no visuales de la luz natural sobre la salud ${ }^{1}$
}

Verónica Giraldo Gaviria ${ }^{1}$ | Lucas Arango Díaz ${ }^{2}$ | Elisabeth Herreño Telléz ${ }^{3}$

Recibido: 20-09-2019 | en su versión final: 09-03-2020

Resumen

El desempeño de la luz natural en ambientes interiores ha sido estudiado en términos de autonomía de la luz y eficiencia energética, siendo común que se excluya el factor humano dentro de las variables de evaluación. No obstante, investigaciones recientes confirman la relevancia de aprovechar la luz natural la mayor parte del día y sus ventajas biológicas para la regulación de los ritmos circadianos; reafirmando la necesidad de incorporar estos conceptos en el diseño arquitectónico. En este artículo se propone una curva de ponderación para estimar el nivel lumínico en unidades equivalentes de lux melanópicos (EML) para diferentes edades, a partir del $98.5 \%$ de supresión de melatonina, adicionalmente, se explora un método de evaluación con simulaciones computacionales de un espacio de oficina y diversas modificaciones arquitectónicas, para usuarios entre 25 y 55 años, utilizando un archivo climático del lugar y una métrica de Autonomía de luz natural (DA). Los resultados obtenidos se compararon con los conceptos de iluminación circadiana de la Certificación WELL y los estudios de Kyle Konis, exponiendo una perspectiva diferente de la valoración del desempeño lumínico y los efectos de salud en ambientes de oficina según la edad. Como conclusión, se evidencia que es posible incorporar un método descriptivo cómo herramienta para ampliar el panorama en análisis más completos de la luz natural en ambientes interiores, dando pistas de la funcionalidad espacial en términos de bienestar, salud y comodidad. Sin embargo, todavía hacen falta avances en las métricas de factores humanos y en herramientas de simulación computacional que faciliten evaluar efectos de salud en la arquitectura.

Palabras clave: factores humanos; iluminación circadiana; ipRGCs; melatonina

Citación

\author{
Giraldo, V. et al (2020). Valoración del desempeño lumínico de puestos de trabajo a partir \\ de los efectos no visuales de la luz natural sobre la salud. ACE: Architecture, City and \\ Environment, 15(43), 8903. DOI: http://dx.doi.org/10.5821/ace.15.43.8903
}

\begin{abstract}
1 Arquitecta, M.Sc. en Bioclimática (ORCID: 0000-0003-1411-9101, WoS Researcher ID: AAR-9140-2020), ${ }^{2}$ Arquitecto, M. Sc., Docente Investigador del Grupo Hombre, Proyecto, Ciudad, Facultad de Artes Integradas, Universidad San Buenaventura, Medellín, Colombia (ORCID: 0000-0002-3638-3379, WoS Researcher ID: AAR-9187-2020, Scopus Author ID: $\underline{57205170758)}$, ${ }^{3}$ Diseñadora Industrial, M. Sc., Docente Investigadora del Grupo Hombre, Proyecto, Ciudad, Facultad de Artes Integradas, Universidad San Buenaventura, Medellín, Colombia (ORCID: 0000-0003-0205-7767, WoS Researcher ID: AAR-9175-2020). Correo de contacto: veronica.giraldo@tau.usbmed.edu.co
\end{abstract}

\footnotetext{
1 Artículo derivado de tesis inédita: Ensayo metodológico para la evaluación simultánea de suficiencia lumínica, probabilidad de deslumbramiento y efectos no visuales en la salud con iluminación natural en oficinas.
} 


\title{
Assessment of light performance in work environments, based on the non-visual effects of natural light on health
}

\begin{abstract}
The performance of natural light in indoor environments has been studied in terms of daylight autonomy and energy efficiency. The human factor is commonly excluded from the assessment variables. However, recent research confirms the relevance of taking advantage of natural light most of the day and its biological benefits regulating circadian rhythms; which reaffirms the need to incorporate these concepts into architectural design. This article proposes a weighting curve to estimate the light level in Equivalent Melanopic Lux (EML) for different ages, starting at 98.5\% of melatonin suppression. In addition, it explores an evaluation method with computational simulations of office space and various architectural modifications; for users between 25 and 55 years old, using a climate file of the place and a metric of Daylight Autonomy (DA). The results obtained were compared with the concepts of circadian lighting of the WELL Certification and the studies of Kyle Konis, exposing a different perspective on the assessment of light performance and health effects in office environments according to age. This research demonstrates that it is possible to incorporate a descriptive method as a tool to broaden the panorama in more complete analyses of natural light in indoor environments, providing clues to spatial functionality in terms of well-being, health, and comfort. However, progress is still needed in human factor metrics and computational simulation tools to facilitate assessing health effects in architecture.
\end{abstract}

Keywords: human factors; circadian lighting; ipRGCs; melatonin

\section{Introducción}

La iluminación natural en la arquitectura es un tema ampliamente estudiado desde diversos enfoques: eficiencia energética (Boyce, Hunter, \& Howlett, 2003; ICONTEC, 2010; IESNA, 2000; Wymelenberg et al., 2010), comodidad visual y deslumbramiento (Arango, 2016; Giraldo \& Arango, 2015; Jakubiec \& Reinhart, 2010), métricas para estimar el nivel lumínico y efectos no-visuales de la luz natural (Andersen et al., 2008; Lucas et al., 2014; Pattini, 2006; Yáñez, 2008). Este último enfoque, relativamente novedoso, ha ido tomando fuerza debido a las implicaciones de la luz sobre la salud de las personas (Andersen, 2015). De hecho, la exposición a la luz natural podría representar cambios en el rendimiento laboral o académico de las personas, mejoras en la sensación de cansancio, fatiga visual y exceso de sueño o falta del mismo (Maria Amundadottir, Rockcastle, Khanie, \& Andersen, 2017; Andersen, 2015; Velux Group, 2010). No obstante, los avances en este último tema evidencian dificultades en el cálculo de la respuesta no-visual de los seres humanos en su relación con el medio, debido a que existen variadas características humanas que determinan las sensibilidades visuales y los estímulos internos; solo hasta el momento unos cuantos efectos propios de la salud cuentan con cantidades medibles (Bará \& Escofet, 2018; Parsons, 2000).

A pesar de esto, en la actualidad se ha trabajado en diferentes métodos de análisis de luz natural en la arquitectura, por ejemplo: Marilyne Andersen (2015) ha estudiado la optimización de la luz del día y el confort visual proponiendo herramientas de diseño que permitan visualizar las implicaciones espaciales y los efectos de la luz sobre los ritmos circadianos y la salud. El arquitecto Christopher Meek (Meek \& Wymelenberg, 2015), ha desarrollado métricas para evaluar el desempeño ambiental de los edificios, específicamente en temas de iluminación ha investigado la disponibilidad de luz diurna anual con modelos computacionales, considerando el comportamiento de los usuarios; y a 
nivel normativo, la Comisión Internacional de la Iluminación (CIE TN 003:2015, 2015) en el 2013 realizó un primer taller internacional sobre fotometría circadiana y neurofisiología, que tenía como objetivo proporcionar una interpretación para los gobiernos, los profesionales en salud pública e iluminación sobre la importancia de los avances científicos en temas relacionados con los espectros lumínicos y los efectos fotobiológicos de la luz que no forma imágenes. En resumen, las investigaciones recientes y la pertinencia de los nuevos descubrimientos en los fotorreceptores amplían el interés no solo a la luz visible que genera imágenes sino también a los efectos no visuales en la salud.

\subsection{Los fotorreceptores}

Los fotorreceptores, o células neuronales sensibles a la luz natural, son de tres tipos: los conos y los bastones que regulan los efectos visuales y las células ganglionares fotosensitivas (ipRGCs) que contienen un fotopigmento llamado melanopsina, encargada de regular los efectos biológicos (Alkozi, 2019; Domínguez \& Pérez, 2015; Lucas et al., 2014; Peréz \& Brown, 2009; Van Bommel \& Van Den Beld, 2004). Los tres fotorreceptores se conectan por el nervio óptico a diferentes partes del cerebro y son responsables de identificar el color y la sensibilidad a la cantidad de luz. Los conos tienen como función la visión a color, los bastones están encargados de la visión con niveles bajos de luz, mientras que los ipRGCs no hacen parte de la generación de imágenes. Estos generan estímulos lentos, señalando la presencia de luz a largo plazo y son los encargados de sincronizar los ciclos circadianos en un periodo de 24 horas, regular el tamaño de la pupila y otros comportamientos relacionados con las condiciones ambientales de iluminación (Bommel \& van den Beld, 2004; Brainard et al., 2001).

\subsection{Avances en los estudios de eficiencia espectral de los ipRGCS - EML}

Desde el 2011 la eficiencia espectral de los ipRGCs ha sido estudiada por Jazi Al. Enezi y Robert J. Lucas (Al Enezi et al., 2011), donde exponen la función de rendimiento melanópico (C-lambda) medido en unidades equivalentes de lux melanópicos o EML, datos que surgen a partir de una caja de herramientas donde cada espectro deseado derivaba en los lux equivalentes de cada fotorreceptor. Esto es aplicable a varias fuentes de luz según el potencial de distribución espectral (SPD). Lo anterior se evidencia en la Figura 1, en la que se comparan SPD de tres iluminantes de luz natural de la CIE (D55/luz solar-D65/cielo cubierto-D75 luz natural hemisferio norte) con la curva de eficiencia fotópica ( $v$-lambda) y circadiana (c-lambda). Esta alineación entre la máxima efectividad circadiana con el máximo potencial de los tres iluminantes es bastante distante de la función visual fotópica ( $V$ Lambda), es decir, la percepción visual que se produce con niveles de iluminación diurnos, posibilitando la interpretación del color. (Al Enezi et al., 2011; Bará \& Escofet, 2017, 2018; D. Galadí, 2018; Lucas et al., 2013).

Figura 1. Comparación de la potencia espectral relativa con tres iluminantes D55-D65-D75

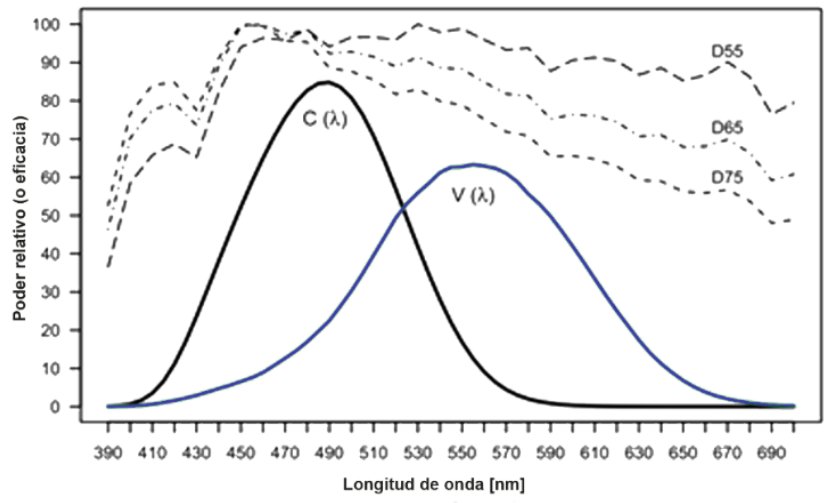

Fuente: (Konis, 2017, pp. 23-24)

ACE, 15 (43) CC BY-ND 3.0 ES | UPC Barcelona, España | Valoración del desempeño lumínico de puestos de trabajo a 
Con relación a la sincronización de los ciclos circadianos, a través de los ipRGCs, nuestro órgano visual codifica y transmite información a partir de la abundancia de la luz del día facilitando la producción de serotonina, responsable del estado de alerta, la supresión de melatonina², también llamada la hormona del sueño y la activación de la producción de cortisol relacionada con el nivel de actividad cerebral y motriz. Cuando los ojos captan los niveles de luz necesarios, el cerebro recibe impulsos relacionados con las emociones y las funciones hormonales (Boubekri, 2014a).

Asimismo, en el hipotálamo, parte del sistema endocrino ubicado en el cerebro, se generan los procesos químicos y físicos del metabolismo y de todas las reacciones químicas de cada célula del cuerpo, siendo responsable de procesos de energía, balance de fluidos, crecimiento, maduración, circulación, respiración, balance de emociones, reproducción, regulación de la temperatura, actividad y patrones de sueño (Boubekri, 2014a). Algunas enfermedades o afectaciones reconocidas por la medicina como alteraciones relacionadas con el fenómeno de la luz son: el trastorno afectivo estacional (SAD), epilepsia fotosensitiva, fatiga ocular, jet lag, dolores de cabeza (Kopec, 2017).

Investigaciones recientes (Amundadottir et al., 2017; Andersen, 2015) han identificado que estimular la melanopsina, un fotopigmento que se encuentra en las células ganglionares fotosensibles de la retina y que están involucradas con la regulación del ritmo circadiano, los reflejos pupilares y las respuestas no visuales a la luz se logra con cinco factores de exposición a la luz, bajo iluminancia vertical. Estos cinco factores son I) intensidad del nivel lumínico, II) longitud de onda de la luz al que se está expuesto, III) duración de exposición a los niveles lumínicos, IV) sincronización del ciclo circadiano y $\mathrm{V}$ ) adaptación al nivel lumínico o exposición previa (Andersen et al., 2008). Algunos avances en estos temas se evidencian en las herramientas de simulación Lightsolve y Spektro que evalúan el desempeño lumínico y las respuestas no visuales (Amundadottir et al., 2014; Rockcastle \& Andersen, 2013).

\subsection{Iluminación circadiana - EML}

Al sistema de iluminación que, a partir de los efectos no-visuales alteran el comportamiento biológico, fisiológico y psicológico y que, por tanto, afectan los ciclos circadianos se le conoce como iluminación circadiana. Un concepto que involucra la luz natural, la arquitectura, los factores humanos y la salud. (International WELL Building Institute, 2016; Rea \& Figueiro, 2016a).

La iluminación circadiana parte del ciclo interno de un ser humano sano, es decir, 24.2 h para mantener el arranque local, 24 horas luz/oscuridad, sin embargo, los efectos producidos en el cuerpo por la luz natural no son instantáneos (Konis, 2017). Estos dependen en gran medida de la cantidad y calidad de la luz que reciba el órgano visual, lo que posteriormente puede evaluarse en bienestar, atención, calidad de sueño, entre otros estímulos.

En la actualidad no se cuenta con normativa para reglamentar la cantidad de lux mínima que apoye el arrastre circadiano, o el tiempo mínimo de exposición a niveles lumínicos que favorezca la supresión de melatonina en los espacios interiores, sin embargo, existen tres referentes para evaluar la dosis de respuesta en la unidad equivalente de lux melanópicos (EML) con relación al porcentaje de supresión de melatonina:

- Mohamed Boubekri en el artículo "Daylighting Design: Planning strategies and best practice solutions" (Boubekri, 2014b) expone que un observador de 65 años necesita de manera progresiva de 77 a 250 EML para suprimir 50 a 98.5\% respectivamente, es decir de 69.6 a 226 lux. En la Figura 2, se observa la relación entre la exposición de luz y los porcentajes de supresión de melatonina,

\footnotetext{
${ }^{2}$ Neurohormona controlada con la activación de melanopsina
} 
en este caso, con una fuente lumínica D65-cielo cubierto: luz día - luz natural, encontrando en la curva que la dosis de luz no es lineal y puede variar según el tipo de fuente lumínica ya sea: (incandescente CIE A-2856k, fluorescente CIE F11-4000k, D65, luz azul LED95-9500k) (Boubekri, 2014b). Ver la Tabla 1, en la que se compara el EML con varias fuentes de luz para un observador de 65 años a 9500K.

Figura 2. Curva de dosis de respuesta - Impacto biológico - Fuente lumínica D65 - Observador de 65 años

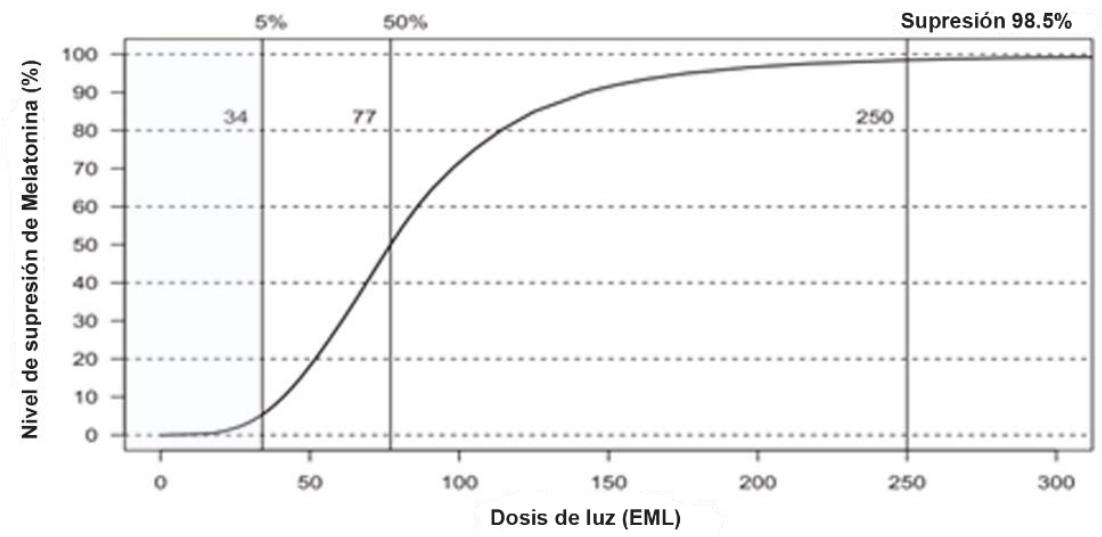

Fuente: (Konis, 2017, pp. 26)

Tabla 1. Impacto biológico para varias fuentes de luz e iluminancias fotópicas

\begin{tabular}{|c|c|c|c|c|c|}
\hline $\begin{array}{c}\text { Supresión de } \\
\text { Melatonina (\%) }\end{array}$ & EML & A (Lux) & F11 (Lux) & D65 (Lux) & LED95 (Lux) \\
\hline 0.5 & 17 & 29 & 27 & 16 & 27 \\
\hline 5.0 & 34 & 56 & 52 & 31 & 52 \\
\hline 25.0 & 56 & 95 & 87 & 71 & 62 \\
\hline 50.0 & 77 & 129 & 118 & 97 & 84 \\
\hline 75.0 & 105 & 176 & 272 & 162 & 142 \\
\hline 95.0 & 176 & 296 & 526 & 315 & \\
\hline 99.5 & 341 & 575 & & & 275 \\
\hline
\end{tabular}

Fuente: (Konis, 2017, pp. 2)

- El International Well Building Institute (International WELL Building Institute, 2016) desarrolló un sistema de certificación con estándares de evaluación de la iluminación circadiana aplicando la métrica del EML propuesta por Robert J. Lucas y otros autores (2014).

Este método simplificado habla de un mínimo de 250 EML para personas entre 25 y 65 años por 4 horas cada día del año, entre las 12:00 m. y 4:00 p.m., medidos en la superficie vertical a la altura del observador (1.20 mts. del piso); cumpliendo con una supresión de melatonina del 98.5\%.

Adicionalmente, la certificación WELL especifica que para calcular el EML en diferentes fuentes de luz, se multiplican los lux visibles ( $L$ ) diseñados o medidos en el edificio por el ratio (R) de la fuente de luz. Fórmula: EML=LXR. En la tabla 2 aparecen los ratios melanopicos para diferentes fuentes lumínicas, según la cantidad de CCT (K). 
Tabla 2. Ratios melanópicos para diferentes fuentes de luz

\begin{tabular}{|l|l|l|}
\hline \multicolumn{3}{|c|}{ Ratios Melanópicos } \\
\hline \multicolumn{1}{|c|}{ CCT $(k)$} & \multicolumn{1}{|c|}{ Fuente de luz } & Ratio \\
\hline \hline 2700 & LED & 0.45 \\
\hline 3000 & Fluorescente & 0.45 \\
\hline 2800 & Incandescente & 0.54 \\
\hline 4000 & Fluorescente & 0.58 \\
\hline 4000 & LED & 0.76 \\
\hline 5450 & ClE E (Energía igual) & 1.00 \\
\hline 6500 & Fluorescente & 1.02 \\
\hline 6500 & Luz día - Luz natural & 1.10 \\
\hline 7500 & Fluorescente & 1.11 \\
\hline \multicolumn{2}{|c|}{ CCT (k): Temperatura de color correlacionada } \\
\hline
\end{tabular}

Fuente: (Elaboración a partir del Internacional WELL Building Institute, 2016, pp. 194)

- En el 2017 Kyle Konis en el artículo "A novel circadian daylight metric for building design and evaluation" (Konis, 2017) propone un método para evaluar los efectos no visuales de la luz, a partir de una métrica de iluminación natural que involucra los estímulos circadianos y el arrastre de los edificios. Además, hace una comparación de la cantidad de luz natural para tres edades de la siguiente manera: un observador de 65 años requiere una intensidad de estímulo $6 \%$ mayor que una persona de 32 años o una intensidad 11\% mayor con respecto a un niño de 10 años, siendo la edad y el horario de trabajo factores adicionales que muchas veces no están directamente relacionados con el diseño de un edificio.

De igual forma, expone que los ritmos circadianos son autosostenibles, logrando persistir en el cuerpo durante un periodo de tiempo significativo, por lo tanto, es posible contar con un arrastre circadiano en los puestos de trabajo a pesar de que no se cuente con el estímulo necesario para un día. Lo importante es identificar a partir de una métrica la efectividad de la luz del día para el estímulo, ejemplo: 4 horas al día, 3650 horas ocupadas al año (Konis, 2017). De igual forma, Konis enfatiza que actualmente el desarrollo de una métrica circadiana para la luz natural se basa en una combinación de información científica y juicios de expertos, que deben ser revisados a medida que evolucione la comprensión científica acerca de la respuesta no-visual a la luz natural o artificial.

Retomando lo anterior, este artículo tiene por objetivo explorar la incorporación de criterios que involucran factores humanos en el análisis de desempeño de la luz natural en ambientes interiores, siendo una parte del resultado de un proyecto de investigación de maestría titulado: Ensayo metodológico para la evaluación simultánea de suficiencia lumínica, probabilidad de deslumbramiento y efectos no visuales en la salud con iluminación natural en oficinas, en el que fueron implementados conceptos de la Certificación WELL y de Kyle Konis (Internacional WELL Building Institute, 2016; Konis, 2017) para procurar evidenciar, de manera cuantitativa, la importancia del diseño de ambientes laborales o educativos sobre la salud de las personas.

\section{Metodología}

La metodología aplicada se divide en dos etapas: la primera etapa describe la propuesta de modificación de la métrica EML, llamada curva de ponderación, para estimar el nivel lumínico en el que se da la supresión de melatonina al 98,5\%; y la segunda etapa, presenta la aplicación del método de evaluación en un caso real a partir de diversas modificaciones arquitectónicas. partir de los efectos no visuales de la luz natural sobre la salud. DOI: http://dx.doi.org/10.5821/ace.15.43.8903 


\subsection{Métrica de EML modificada - Etapa 1}

Debido a la novedad del tema y a los vacíos teóricos para la valoración de factores humanos con el uso de la luz natural, se evidencia la falencia de un sistema práctico de medición de EML para diferentes edades y porcentajes de supresión de melatonina. Por tanto, resulta pertinente construir una herramienta con el fin de estimar la cantidad de EML necesarios para la supresión de melatonina en un $98,5 \%$ para edades de 25 y 55 años.

En la construcción de esta herramienta se cruzó información de diferentes fuentes teóricas con el objetivo de relacionar datos que no limiten por edad las valoraciones para la estimación de la iluminación circadiana: en primer lugar se identificó que 250 EML corresponden a 226 lux según el (Internacional WELL Building Institute, 2016), dato importante para convertir a partir de una regla de tres los valores equivalentes de EML a lux, una unidad de medida reconocida actualmente por los programas computacionales que simulan iluminación natural. Posteriormente, con el fin identificar los EML necesarios para los usuarios entre 25 y 55 años, edades correspondientes al caso real simulado, se graficó en la Figura 3 la curva de ponderación.

Figura 3. Curva de ponderación del $98.5 \%$ de supresión de melatonina según la edad y la dosis de luz

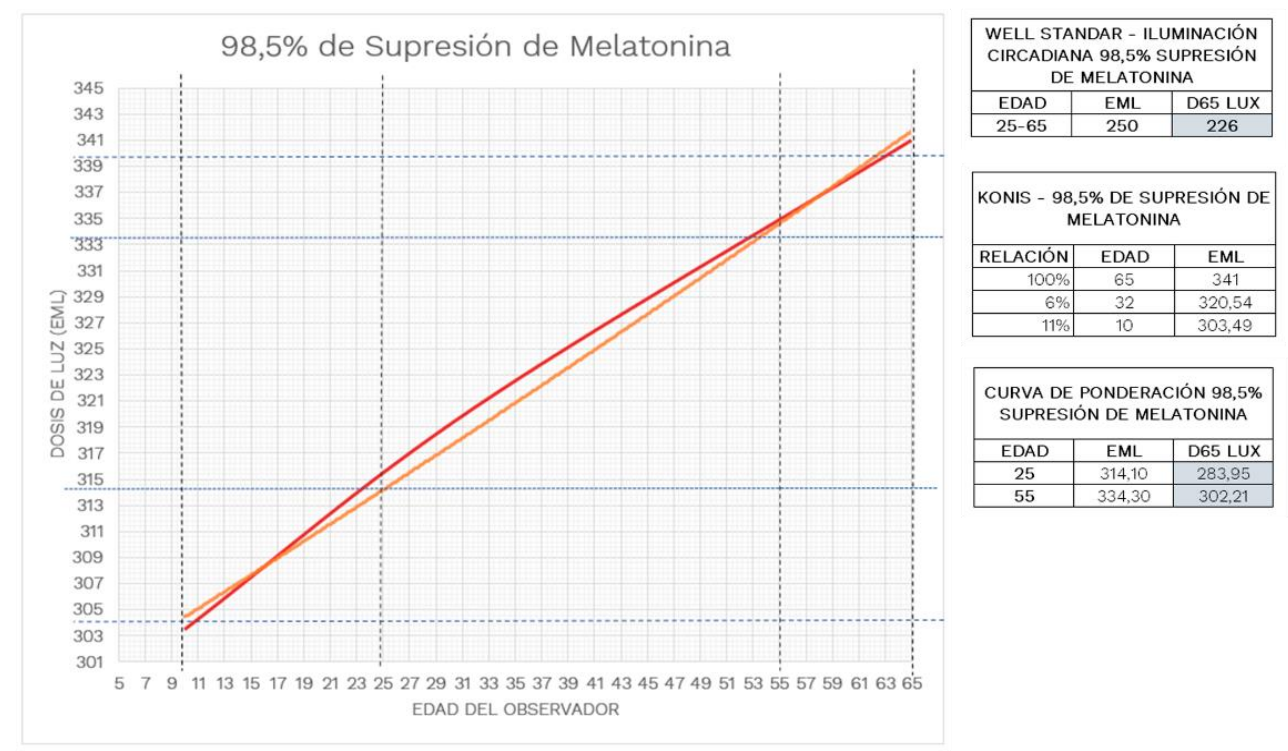

Fuente: Elaboración propia a partir del Internacional WELL Building Institute (2016, pp. 96) y Konis (2017, pp. 24)

Esta herramienta, planteada con los estándares de certificación WELL y los estudios de Kyle Konis, se construyó con unos valores cercanos de EML para usuarios de 10 a 65 años, donde se visualiza específicamente que una persona de 25 años requiere 314,10 EML (283,95lux) y una de 55 años necesita 334,30 EML (302.21 lux) para suprimir un 98,5\% de melatonina.

Como conclusión de la información estudiada y graficada, se encuentra que la unidad equivalente de lux melanópicos (EML) para suprimir el 98.5\% de melatonina no es lineal entre edades, incluso por que dependen del tipo de fuente o iluminante CIE entre otros (Konis, 2017; M. S. Rea, Figueiro, Bierman, \& Hamner, 2012). Para el caso de este trabajo, la curva de ponderación construida a partir de la relación entre la información propuesta por la certificación WELL (Internacional WELL Building Institute, 2016) y la investigación de Kyle Konis (Konis, 2017) da indicios de valores comparables entre EML y lux para edades entre 10 y 65 años, ampliando las posibilidades de conocer la cantidad de iluminación circadiana en espacios de trabajo existentes o hipotéticos, diferenciando la necesidad lumínica según la edad de los usuarios. 


\subsection{Aplicación del método de evaluación - Etapa 2}

Una vez realizada la propuesta de modificación de EML para diferentes edades, se procedió a ensayar la propuesta metodológica de evaluación de los efectos no-visuales en la salud para un espacio de trabajo existente. En este caso se seleccionó una oficina localizada en la ciudad de Medellín, Colombia (lat. 6,25, lon. -75,5, alt. 1.495 m.s.n.m.), de geometría rectangular y una distribución espacial de planta libre (Figura 4), con una sola ventana al sur, seis lucernarios clausurados y acabados claros y oscuros en las superficies interiores.

Figura 4. Espacio de trabajo seleccionado

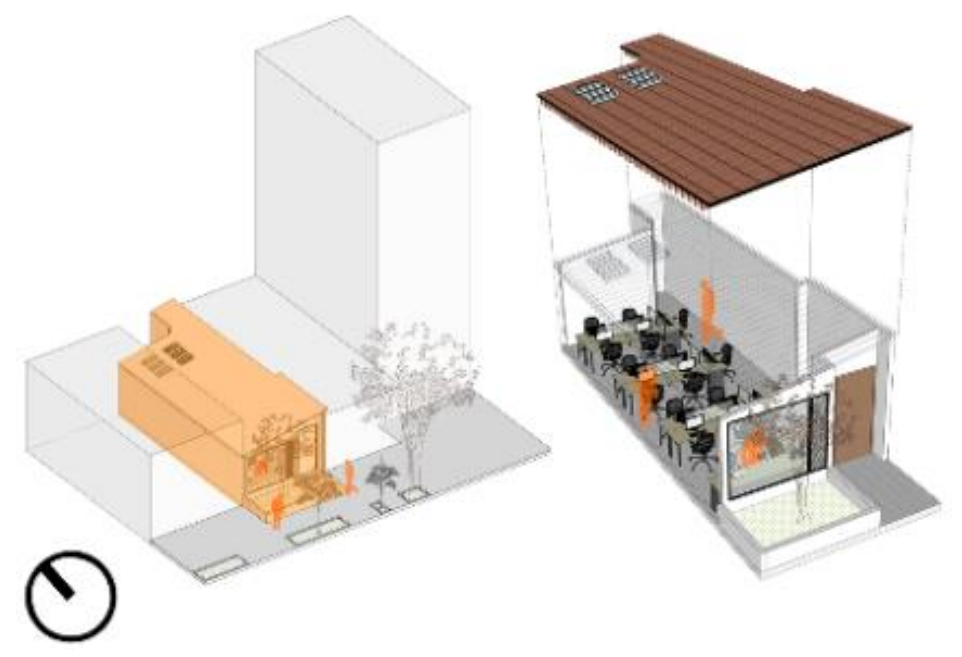

Fuente: (Elaboración propia)

En el programa computacional Rhino con el plug-in Diva, se modelaron siete puestos de trabajo (ver figura 5) en el ambiente evaluado y fueron realizadas simulaciones dinámicas haciendo uso de la métrica Daylight Autonomy - DA evaluada en el plano vertical a la altura de los ojos (1,2m). Para 25 años se usó una iluminancia objetivo de 283,95lux, mientras que para las simulaciones con personas de 55 años se usó 302.21 lux.

Figura 5. Puestos de trabajo evaluados en el caso real

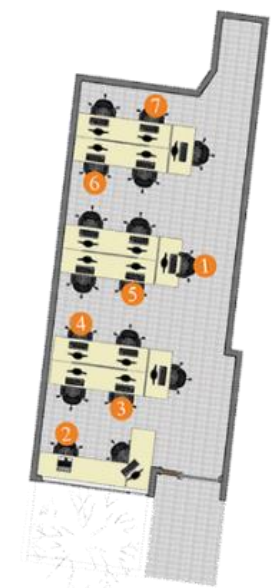

(T) Planta libre $53.25 \mathrm{~m} 2$ - Altura libre $3.0 \mathrm{mt}$

Ventana sur 2.0x3.0 mt - Lucernarios (6) $1.0 \times .40 \mathrm{mt}$

Fuente: (Elaboración propia)

ACE, 15 (43) CC BY-ND 3.0 ES | UPC Barcelona, España | Valoración del desempeño lumínico de puestos de trabajo a partir de los efectos no visuales de la luz natural sobre la salud. DOI: http://dx.doi.org/10.5821/ace.15.43.8903 
En el programa fueron considerados los parámetros de simulación descritos en la Tabla 3.

Tabla 3. Parámetros de simulación de diva

\begin{tabular}{|c|c|c|c|c|}
\hline Ambient Bounces & Ambient Division & Ambient Sampling & Ambient Resolution & Ambient Accuracy \\
\hline 7 & 1000 & 20 & 300 & 0,1 \\
\hline
\end{tabular}

Fuente: (Elaboración propia)

Se utilizó un archivo climático de la zona de análisis, con una programación anual e intervalos de 60 minutos entre las 08h-00 min. a las 18h-00 min., considerando durante este tiempo solo el uso de luz natural sin dispositivos de protección solar en las ventanas.

Tabla 4. Propiedades ópticas de los materiales

\begin{tabular}{|c|c|c|c|c|c|c|c|c|}
\hline \multicolumn{9}{|c|}{ CARACTERÍSTICAS ESPACIALES DE LA OFICINA } \\
\hline \multirow{2}{*}{$\begin{array}{c}\text { CAPAS } \\
\text { Propiedades }\end{array}$} & \multicolumn{2}{|c|}{ CR-MTM 01-7 } & \multicolumn{2}{|c|}{ CMA-MTM01-7 } & \multicolumn{2}{|c|}{ CMB-MTM01-7 } & \multicolumn{2}{|c|}{ CMC-MTM01-7 } \\
\hline & $\% \mathrm{R}$ & $\%(\mathrm{TN})$ & $\% \mathrm{R}$ & $\%(\mathrm{TN})$ & $\% \mathrm{R}$ & $\%(\mathrm{TN})$ & $\% \mathrm{R}$ & $\%(\mathrm{TN})$ \\
\hline Anden & 30 & & 30 & & 30 & & 30 & \\
\hline Árboles & 35 & & 35 & & 35 & & 35 & \\
\hline Cubierta & 20 & & 20 & & 70 & & 20 & \\
\hline Lucernarios* & 20 & & & 20 & & 20 & & 20 \\
\hline Muros & 70 & & 70 & & 70 & & 40 & \\
\hline Muros bajos & 30 & & 30 & & 30 & & 30 & \\
\hline Paneles laterales en vidrio** & & 20 & & 20 & & 20 & & 20 \\
\hline Pantallas & 30 & & 30 & & 30 & & 30 & \\
\hline Pisos & 40 & & 40 & & 70 & & 40 & \\
\hline Puertas en madera & 30 & & 30 & & 50 & & 20 & \\
\hline Superficies de trabajo & 50 & & 50 & & 70 & & 30 & \\
\hline Vidrio & & 88 & & 58 & & 88 & & 88 \\
\hline Zonas verdes & 35 & & 35 & & 35 & & 35 & \\
\hline
\end{tabular}

Fuente: (Elaboración propia)

Con el fin de identificar la mayor o menor influencia de las propiedades ópticas de los materiales sobre la supresión de melatonina, a partir del espacio modelado se realizaron las simulaciones de un caso real (CRMTM01-7), haciendo uso de los coeficientes de reflectancia o transmitancia de las superficies según los materiales reales, y tres casos modificados con los materiales (descritos en la Tabla 4): CMAMTM01-7-cambio en superficies de vidrio, CMBMTM01-7-superficies claras y CMCMTM01-7-superficies oscuras.

\subsubsection{Análisis de los puestos de trabajo}

Para evaluar los efectos de la luz natural en la salud según el puesto de trabajo y el uso de la métrica EML modificado para diferentes edades, se definió una tabla de valoración según el porcentaje de tiempo al año en que la iluminancia vertical a la altura de los ojos superaba el valor necesario para garantizar supresión de melatonina. Estos porcentajes se distribuyeron en rangos (BUENO MODERADO - NO DESEADO) como se muestra en la tabla 5. 
Tabla 5. Rangos de evaluación de desempeño según la variable salud por efectos no visuales

\begin{tabular}{|c|c|c|c|}
\hline No deseado & Moderado & Bueno \\
\hline $0,0 \%-22,4 \%$ & $22,5 \%-44,9 \%$ & $45,0 \%-100,0 \%$ \\
\hline
\end{tabular}

Fuente: (Elaboración propia)

Estos rangos fueron estimados a partir del arrastre circadiano que corresponde a más de 4 horas por encima del nivel lumínico a las que una persona debe exponerse por cada día del año para suprimir el $98.5 \%$ de melatonina. El bueno se encuentra entre 45\%-100\%, es decir, más de 4 horas, el moderado entre $22,5 \%$ - 44,9\% que corresponde a 2-4 horas de exposición a iluminancias verticales mientras que el no deseado, 0\%-22,4\%- está por debajo de 2 horas.

\section{Resultados}

A continuación, se exponen los resultados de las simulaciones computacionales realizadas para la oficina. Para cada puesto de trabajo se identificó la influencia de las modificaciones en las propiedades ópticas de los materiales sobre la iluminancia vertical medida en SDA. En la tabla 6, se muestran los valores de Daylight Autonomy - DA de las simulaciones del caso real (CRMTM01-7) y las tres modificaciones para siete puestos de trabajo. Adicionalmente, en la tabla se resaltan con colores los casos en los que se obtuvo iluminación circadiana buena, moderada o no deseada.

Tabla 6. Datos de simulaciones de CR/CMA/CMB/CMC para la salud por efectos no visuales en usuarios de 25 y 55 años

\begin{tabular}{|c|c|c|c|c|c|c|c|}
\hline \multicolumn{8}{|c|}{ Casos simulados - 25 años } \\
\hline Puestos de trabajo & PT 1 & PT 2 & PT 3 & PT 4 & PT 5 & PT 6 & PT 7 \\
\hline CRMTM01-7 & $4,00 \%$ & $99,00 \%$ & $69,00 \%$ & $46,25 \%$ & $1,00 \%$ & $0,00 \%$ & $0,00 \%$ \\
\hline CMAMTM01-7 & $17,50 \%$ & $98,00 \%$ & $41,75 \%$ & $20,25 \%$ & $12,75 \%$ & $2,00 \%$ & $7,00 \%$ \\
\hline CMBMTM01-7 & $50,75 \%$ & $99,00 \%$ & $82,50 \%$ & $70,75 \%$ & $33,50 \%$ & $2,75 \%$ & $14,75 \%$ \\
\hline СMCMTM01-7 & $24,50 \%$ & $98,75 \%$ & $56,50 \%$ & $31,50 \%$ & $10,75 \%$ & $1,00 \%$ & $6,25 \%$ \\
\hline \multicolumn{8}{|c|}{ Casos simulados - 55 años } \\
\hline Puestos de trabajo & PT 1 & PT 2 & PT 3 & PT 4 & PT 5 & PT 6 & PT 7 \\
\hline CRMTM01-7 & $4,00 \%$ & $98,75 \%$ & $66,55 \%$ & $41,50 \%$ & $1,00 \%$ & $0,00 \%$ & $0,00 \%$ \\
\hline CMAMTM01-7 & $14,00 \%$ & $98,00 \%$ & $36,75 \%$ & $16,25 \%$ & $10,00 \%$ & $2,00 \%$ & $6,75 \%$ \\
\hline CMBMTM01-7 & $47,50 \%$ & $99,00 \%$ & $81,25 \%$ & $70,50 \%$ & $31,75 \%$ & $2,25 \%$ & $14,00 \%$ \\
\hline СMCMTM01-7 & $20,50 \%$ & $98,75 \%$ & $52,50 \%$ & $28,50 \%$ & $9,00 \%$ & $1,00 \%$ & $7,75 \%$ \\
\hline No deseado & \multicolumn{2}{|c|}{ Moderado } & \multicolumn{2}{|c|}{ Bueno } & & & \\
\hline
\end{tabular}

Fuente: (Elaboración propia

Los resultados evidencian un comportamiento muy similar para las personas de 25 y 55 años, además de mostrar que los puestos de trabajo 1, 5, 6 y 7 en el caso real poseen la mayor parte del año EML inferiores a los recomendados para garantizar la supresión de melatonina. El puesto de trabajo 2, en todos los casos, por estar tan próximo a la ventana, muestra que gran parte del año posee niveles lumínicos superiores a lo necesario para la supresión de melatonina. Esta sobreexposición podría resultar inconveniente desde el punto de vista del confort y de salud por efectos no-visuales. El puesto de trabajo 3 en los casos CR/CMB/CMC cumple el rango bueno para temas de salud, condición 
favorable por su ubicación no mayor a 2 mts de la ventana. Y el puesto 4, resulta ser bueno para la persona de 25 años en el caso CR/CMB y moderado en el caso CR/CMC para la de 55 años. En resumen, el estado actual de la oficina evaluada (CRMTM01-7) para 25 años, cuenta con tres casos en un rango bueno y cuatro en un rango no deseado, y para 55 años, tres casos en rango bueno, un caso en moderado y tres casos en no deseado.

Con relación a la influencia de las propiedades ópticas de los materiales se resalta la alta influencia del color de las superficies, en tanto que el caso CMBMTM01-7, que poseía superficies claras al interior, mejoró notablemente el desempeño anual del EML en la mayoría de los puestos de trabajo. De hecho, el libro Daylighting and Integrated lighting Design afirma cómo las superficies interiores según su color y acabado sirven para reflejar la luz, reducir los contrastes, controlar los brillos molestos y alcanzar los niveles de lux deseados de manera homogénea en todo el espacio (Meek \& Wymelenberg, 2015). De igual forma otros artículos concluyen la efectividad de la iluminación interior mediante el uso de la luz cenital a partir de tragaluces con superficies translucidas o transparentes (Novljan \& Rihtar, 2015).

\section{Conclusiones}

Durante el desarrollo analítico y práctico del método de evaluación, se concluye que la exploración de criterios que involucran factores humanos en el análisis del desempeño de la luz natural, debe considerar lo siguiente:

\subsection{Métrica de EML}

Estandarizar una medida de EML o lux para un rango de edad en temas de evaluación de efectos novisuales, invalida la variación por edad o factores de salud. De hecho, el método simplificado de la Certificación WELL acepta 250 EML entre 25 y 65 años, para suprimir un 98.5\% de melatonina, un valor que se encuentra por debajo de la necesidad de EML para una edad en específico. Es decir, 25 años necesita 314 EML, 55 años 334 EML y 65 años 342 EML. Datos resultantes de la curva de ponderación (Figura 3), construida en este trabajo como consecuencia de la falta de información métrica de luz natural en unidades de EML, equivalente para otras edades.

\subsection{Desempeño de la variable luz natural}

Se identificó que, a mayor admisión de luz en el transcurso del día, mayores son las posibilidades de cumplir los rangos mínimos de EML. Sin embargo, excederse en la dosis de luz aumenta los valores de EML generando incomodidad visual y sobrepasando la cantidad de horas efectivas, lo cual descontrola los ciclos circadianos. En otras palabras, las consecuencias de excesos de luz (natural/artificial) conllevan a activaciones adicionales de estados de alerta, disminución en el sueño, desequilibrio psicológico, fisiológico y biológico en el ser humano.

Según la literatura consultada (Alkozi, 2019; Ávila \& Arias, 2015; Boubekri, 2014a; Brainard et al., 2001; CIE TN 003:2015, 2015; Kopec, 2017), los excesos de luz desequilibran la producción de hormonas de serotonina, melatonina, cortisol, entre otras, principalmente cuando se usa luz artificial, encontrando hoy que la iluminación interior y exterior es un problema de salud pública (AMA, 2013; Mark S Rea \& Figueiro, 2016b). En el caso para la luz natural solo se ha estimado que, a menor cantidad de admisión de luz natural dentro de un espacio, menores son los beneficios de salud en el ser humano.

ACE, 15 (43) CC BY-ND 3.0 ES | UPC Barcelona, España | Valoración del desempeño lumínico de puestos de trabajo a partir de los efectos no visuales de la luz natural sobre la salud. DOI: http://dx.doi.org/10.5821/ace.15.43.8903 


\subsection{De la metodología propuesta}

El ensayo de la metodología de evaluación presentada en este artículo permitió corroborar los datos con una estructura de análisis organizada. Esto demostró la pertinencia de relacionar conceptos de la ciencia, la arquitectura y la salud, con el objetivo de concluir de forma cuantitativa las características físicas y espaciales que intervienen dentro de un proyecto arquitectónico, causando mayor o menor estímulo para efectos de salud. Adicionalmente, el análisis de los espacios de trabajo en temas relacionados con factores humanos concluye que, a mayor equilibrio entre el porcentaje de tiempo con la dosis adecuada de luz para la salud por efectos no-visuales, mayores son las ventajas en términos de bienestar, productividad y salud, marcando una gran diferencia a nivel perceptivo y cognitivo para el ser humano cuando se utiliza luz natural en sus ambientes de trabajo. Ser conscientes de los efectos positivos y negativos que conlleva la iluminación para el ser humano aumentaría el uso de la luz del día mejorando la salud y disminuiría el consumo de energía por el uso de luz artificial en los espacios interiores.

El uso de herramientas de evaluación como la propuesta en este trabajo, facilita a los arquitectos e ingenieros afines a la construcción poder tomar decisiones asertivas en etapas iniciales del diseño arquitectónico o de posibles adecuaciones espaciales, logrando identificar el desempeño lumínico y el aporte en temas de la salud según el uso, la edad y la temporalidad de los ocupantes.

\section{Agradecimientos}

Agradecimientos a la Maestría en Bioclimática de la Universidad San Buenaventura Medellín, Colombia, por su interés y apoyo durante el proceso de evaluación y desarrollo de la tesis de grado.

\section{Autoría}

La investigación, estructuración del trabajo y aplicación de la metodología fue realizado por la primera autora, durante el desarrollo de la tesis de grado de la Maestría en Bioclimática. La tutoría, el proceso de revisión y la complementación de la redacción fue realizada por el segundo y la tercera autora. Todos los autores aportaron, desde de su conocimiento específico, a cada una de las etapas de la investigación, siendo evidente la integralidad de conceptos entorno a los factores humanos y la arquitectura.

Conflicto de intereses: Los autores declaran que no hay conflicto de intereses.

\section{Bibliografía}

Al Enezi, J.; Revell, V.; Brown, T.; Wynne, J.; Schlangen, L., \& Lucas, R. (2011). A "melanopic" spectral efficiency function predicts the sensitivity of melanopsin photoreceptors to polychromatic lights. Journal of Biological Rhythms, 26(4), 314-323. DOI: https://doi.org/10.1177/0748730411409719

Alkozi, H.A. (2019). Melatonin and melanopsin in the eye: friends or foes? - Melatonina y melanopsina en el ojo: ¿amigos o enemigos. Revista Anales de la real academia nacional de farmacia, 85(1), 49-59. Recuperado de: https://analesranf.com/articulo/8501 rev02/

AMA. (2013). AMA adopts new policies on second day of voting at annual meeting. AMA News Room, 1-5. Recuperado de http://www.ama-assn.org/ama/pub/news/news/2013/2013-06-18-new-amapolicies-annual-meeting.page

Amundadottir, M; Lockley, S., \& Andersen, M. (2014). A Unified Framework for Evaluating Non-Visual Spectral Effectiveness of Ocular Light Exposure: Key Concepts. 28th CIE Session.

ACE, 15 (43) CC BY-ND 3.0 ES | UPC Barcelona, España | Valoración del desempeño lumínico de puestos de trabajo a partir de los efectos no visuales de la luz natural sobre la salud. DOI: http://dx.doi.org/10.5821/ace.15.43.8903 
Amundadottir, M.; Rockcastle, S.; Khanie, M., \& Andersen, M. (2017). A human-centric approach to assess daylight in buildings for non-visual health potential, visual interest and gaze behavior. Building and Environment, 113, 5-21. DOI: https://doi.org/10.1016/j.buildenv.2016.09.033

Andersen, M. (2015). Unweaving the human response in daylighting design. Building and Environment, 91, 101-117. DOI: https://doi.org/10.1016/j.buildenv.2015.03.014

Andersen, M.; Kleindienst, S.; Yi, L.; Lee, J.; Bodart, M., \& Cutler, B. (2008a). An intuitive daylighting performance analysis and optimization approach. Building Research and Information, 36(6), 593-607. DOI: https://doi.org/10.1080/09613210802243159

Arango, L. (2016). Deslumbramiento en ambientes educativos con muro calado en fachada. Aus, 20, 62-69. DOI: https://doi.org/10.4206/aus.2016.n20-10

Ávila, D.C., \& Arias, S.A. (2015). La envolvente arquitectónica y su influencia en la iluminación natural. Hábitat Sustentable, 5(1), 44-53. Recuperado de http://revistas.ubiobio.cl/index.php/RHS/article/view/1811

Bará, S., \& Escofet, J. (2017). Research note: Calculating spectral irradiance indoors. Lighting Research and Technology, 49(1), 122-127. DOI: https://doi.org/10.1177/1477153516667643

Bará, S., \& Escofet, J. (2018). On lamps, walls, and eyes: The spectral radiance field and the evaluation of light pollution indoors. Journal of Quantitative Spectroscopy and Radiative Transfer, 205, 267-277. DOI: https://doi.org/10.1016/j.jpsrt.2017.09.022

Bommel, W. van, \& van den Beld, G. van den. (2004). Lighting for work: A review of visual and biological effects. Lighting, Philips, 4, 255-269. DOI: https://doi.org/10.1191/1365782804li122oa

Boubekri, M. (2014a). Daylighting Design: Planning Strategies and Best Practice Solutions. Recuperado de https://books.google.com.co/books?id=C9OBMAEACAAJ

Boubekri, M. (2014b). Daylighting Design: Planning Strategies and Best Practice Solutions. Building Design, p. 176. https://doi.org/10.1515/9783038214786

Boyce, P.R.; Hunter, C., \& Howlett, O. (2003). The Benefits of Daylight through Windows. Capturing the Daylight Dividend Program, 1, 1-88. Recuperado de https://www.semanticscholar.org/paper/TheBenefits-of-Daylight-through-Windows-Boyce-Hunter/99e627e7725f4ffd58792d8ee7a1687dd731ab3c

Brainard, G.C.; Hanifin, J.R.; Greeson, J.M.; Byrne, B.; Glickman, G.; Gerner, E., \& Rollag, M.D. (2001). Action spectrum for melatonin regulation in humans: Evidence for a novel circadian photoreceptor. Journal of Neuroscience, 21(16), 6405-6412. DOI: https://doi.org/10.1523/jneurosci.21-16-06405.2001

CIE TN 003:2015. (2015). Report on the First International Workshop on Circadian and Neurophysiological Photometry, 2013. CIE Technical Report.

D. Galadí, E. (2018). Beyond CCT: The spectral index system as a tool for the objective, quantitative characterization of lamps. Journal of Quantitative Spectroscopy \& Radiative Transfer, 206, 399-408. DOI: https://doi.org/10.1016/j.jgsrt.2017.12.011

Domínguez, C.A., \& Pérez, J.A. (2015). Mecanismo de fototraducción de la melanopsina en las células ganglionares retinianas intrínsecamente fotosensibles (IPRGC). Gaceta Medica de Mexico, 151(6), 764776. Recuperado de https://www.anmm.org.mx/GMM/2015/n6/GMM 15120156 764-776.pdf

Giraldo, N., \& Arango, L. (2015). Propuesta metodológica para la evaluación del desempeño lumínico de fachadas. Arquetipo, (11), 61-76. DOI: https://doi.org/10.31908/22159444.3011

ICONTEC. (2010). NTC 3955- ERGONOMIA.DEFINICIONES Y CONCEPTOS ERGONÓMICOS. 3955. Recuperado de http://www.sociedadcolombianadeergonomia.com/docs/CP DE 063-10.pdf

IESNA. (2000). Lighting Handbook - Illuminating Engineering Society of North America (Ninth; J. Block, Ed.). New York.

ACE, 15 (43) CC BY-ND 3.0 ES | UPC Barcelona, España | Valoración del desempeño lumínico de puestos de trabajo a partir de los efectos no visuales de la luz natural sobre la salud. DOI: http://dx.doi.org/10.5821/ace.15.43.8903 
Internacional WELL Building Institute. (2016). The WELL Building standard (I). New York: Delos Living LLC.

Jakubiec, A., \& Reinhart, C. (2010). The use of glare metrics in the design of daylit spaces: recommendations for practice. 9th International Radiance Workshop, Harvard Design School.

Konis, K. (2017). A novel circadian daylight metric for building design and evaluation. Building and Environment, 113, 22-38. DOI: https://doi.org/10.1016/j.buildenv.2016.11.025

Kopec, D. (2017). Health and Well-being for Interior Architecture. In Routledge \& Edición 1 (Eds.), Health and Well-being for Interior Architecture. DOI: https://doi.org/10.4324/9781315464411

Lucas, R.J.; Peirson, S.N.; Berson, D.; Brown, T.; Cooper, H.; Czeisler, C.A., ... John, B. (2013). Irradiance

Toolbox User Guide. (October), 1-19. Recuperado de

https://personalpages.manchester.ac.uk/staff/robert.lucas/Lucas\%20et\%20al\%202014\%20suppl\%20text.pdf

Lucas, R.J.; Peirson, S.N.; Berson, D.; Brown, T.; Cooper, H.; Czeisler, C.A., ... Brainard, G.C. (2014). Measuring and using light in the melanopsin age. Trends in Neurosciences, 37(1), 1-9. DOI: https://doi.org/10.1016/j.tins.2013.10.004

Meek, C., \& Wymelenberg V. Den., K. (2015). Daylighting and integrated lighting design (Routledge, Ed.). Abingdon, England.

Novljan, T., \& Rihtar, J. (2015). Flat light guide - A sustainable and creative light source. ACE: Architecture, City and Environment, 10(28), 77-90. DOI: https://doi.org/10.5821/ace.10.28.2730

Parsons, K.C. (2000). Environmental ergonomics: A review of principles, methods and models. Applied Ergonomics, 31(6), 581-594. DOI: https://doi.org/10.1016/S0003-6870(00)00044-2

Pattini, A. (2006). Luz natural e iluminación de interiores. Manual de lluminación Eficiente, 2-24.

Peréz, J., \& Brown, R. (2009). Las células con melanopsina: nuevos fotorreceptores en la retina de los vertebrados. REB, 28(1), 9-18. Recuperado de https://www.medigraphic.com/pdfs/revedubio/reb2009/reb091c.pdf

Rea, M.S.; Figueiro, M.G.; Bierman, A., \& Hamner, R. (2012). Modelling the spectral sensitivity of the human circadian system. Lighting Research and Technology, 44(4), 386-396. DOI: https://doi.org/10.1177/1477153511430474

Rea, Mark S., \& Figueiro, M. G. (2016a). Light as a circadian stimulus for architectural lighting. Lighting Research and Technology, 1-14. DOI: https://doi.org/10.1177/1477153516682368

Rea, Mark S, \& Figueiro, M. G. (2016b). Response to the 2016 AMA Report on LED Lighting. AMA, 1-8.

Rockcastle, S., \& Andersen, M. (2013). Annual dynamics of daylight variability and contrast. A Simulation-Based Approach to Quantifying Visual Effects in Architecture. Londres, Reino Unido: Springer. DOI: https://doi.org/10.1007/978-1-4471-5233-0

Van Bommel, W., \& Van Den Beld, G. (2004). Lighting for work: a review of visual and biological effects. Lighting Research \& Technology, 36(4), 255-266. DOI: https://doi.org/10.1191/1365782804li1220a

Velux Group. (2010). Daylight, Energy and Indoor Climate Basic Book. (June), 192.

Wymelenberg V. Den, K.; Inanici, M., \& Johnson, P. (2010). The effect of luminance distribution patterns on occupant preference in a daylit office environment. LEUKOS - Journal of Illuminating Engineering Society of North America, 7(2), 103-122. DOI: https://doi.org/10.1582/LEUKOS.2010.07.02003

Yáñez, G. (2008). Arquitectura solar e iluminación natural. Conceptos, métodos y ejemplos (Munilla Lería, Ed.). 